\title{
The Effects of Caffeine and Pre-Workout Multi- Ingredient Performance Supplement on Reactive Agility and Countermovement Jump Height
}

Piotr Kaczka ( $\square$ kaczor81@o2.pl)

Akademia Wychowania Fizycznego imienia Jerzego Kukuczki w Katowicach https://orcid.org/00000002-1730-6335

\section{Katarzyna Kubicka}

Academy of Physical Education in Katowice

\section{Amit Batra}

Academy of Physical Education in Katowice

\section{Marcin Maciejczyk}

Academy of Physical Education in Cracow: Akademia Wychowania Fizycznego im Bronislawa Czecha

Rafał Jastrąb

Academy of Physical Education in Katowice

\section{Edyta Kopera}

Academy of Physical Education in Katowice

Justyna Bira

Academy of Physical Education in Katowice

Halina Zakliczyńska

Centre of Nutritional Education and Sport

Tomasz Zając

Academy of Physical Education in Katowice

\section{Research article}

Keywords: pre-workout supplementation, agility, countermovement jump, CMJ, caffeine, multi-ingredient performance supplement, MIPS, 1-1-2 reactive agility test, Y-test

Posted Date: January 21st, 2021

DOI: https://doi.org/10.21203/rs.3.rs-150255/v1

License: (c) (i) This work is licensed under a Creative Commons Attribution 4.0 International License.

Read Full License 
1 The Effects of Caffeine and Pre-Workout Multi-Ingredient Performance

2 Supplement on Reactive Agility and Countermovement Jump Height

3 Piotr Kaczka ${ }^{a^{*}}$, Katarzyna Kubicka ${ }^{\mathrm{a}}$, Amit Batra ${ }^{\mathrm{a}}$, Marcin Maciejczyk ${ }^{\mathrm{b}}$, Rafał Jastrząb ${ }^{\mathrm{a}}$,

$4 \quad$ Edyta Kopera ${ }^{\mathrm{a}}$, Justyna Bira ${ }^{\mathrm{a}}$, Halina Zakliczyńska ${ }^{\mathrm{c}}$, Tomasz Zając ${ }^{\mathrm{a}}$

$5 \quad{ }^{a}$ Department of Sport Nutrition, Academy of Physical Education in Katowice, ul.

$6 \quad$ Mikolowska 72a, 40-065 Katowice, Poland

$7{ }^{b}$ Department of Physiology and Biochemistry, University of Physical Education in Krakow,

8 al. Jana Pawła II 78, 31-571 Kraków, Poland

$9{ }^{c}$ Center of Nutritional Education and Sport, ul. Kościuszki 55/2, 40-047 Katowice, Poland

11 * Corresponding author: Piotr Kaczka, e-mail: kaczor81@o2.pl 
Background: The purpose of this investigation was to examine the acute effects of a commercially available multi-ingredient performance supplement (MIPS) in comparison to caffeine, on reactive agility and jump height, in recreationally-trained handball male players.

Methods: The study followed a randomized double-blind, crossover design. Twenty-four individuals were treated with placebo, caffeine or multi-ingredient performance supplement (MIPS). Dosage of caffeine in caffeine supplement and MIPS contain the same amounts of caffeine for each subject $(5 \mathrm{mg} / \mathrm{kg})$. All subjects attended a familiarization session 1 week before testing. Participants underwent reactive agility test (Y-test: 1-1-2 test) on three separate days with $72 \mathrm{~h}$ apart between test days. Just after accomplishing of each repetition of reactive agility tests participants were asked to perform countermovement jump (CMJ; 2 repetitions separated by one minute break) to establish the average height of jump, that is directly related to lower body power. To assess the significance of differences between the groups studied, Friedmann ANOVA test was used, the chi-square value $\left(\chi^{2}\right)$ calculated and the p value of statistical significance estimated.

Results: The research indicated significant inter-group differences in 1-1-2 agility test $(\mathrm{p}=0.04)$. The time was significantly shorter for MIPS supplement group in comparison to placebo ($3.4 \% ; \mathrm{p}=0.02)$ and to caffeine $(-2.8 \% ; \mathrm{p}=0.004)$ group. Significant improvements $(\mathrm{p}<0.01)$ were observed in jump height for MIPS supplement group in comparison to placebo $(+5.2 \%$; $\mathrm{p}=0.001)$ and caffeine $(+5.1 \% ; \mathrm{p}=0.001)$ groups, but not in caffeine to placebo comparison $(\mathrm{p}=0.84)$

Conclusions: The results of these study may contribute to the assessment of the usefulness of multi-ingredient performance type supplementation in comparison to the ingestion of identical amounts of caffeine only in enhancing reactive agility and jump performance. 
Keywords: pre-workout supplementation, agility, countermovement jump, CMJ, caffeine, multi-ingredient performance supplement, MIPS, 1-1-2 reactive agility test, Y-test.

\section{Background}

Agility is a skill required for most field sports. Sheppard and Young (1) have defined reactive agility as a rapid whole body movement with change of velocity or direction in response to a stimulus. In many sport disciplines, changes of speed or rapid and decisive changes of direction can result in a break, a score or a shift in the momentum of the game (2). It should be noted that successful performance in team sports stresses not only physiological systems but also imposes a high degree of technical and cognitive demands (3). Therefore methods effecting in improving psychomotor performance is gaining importance among players, coaches and sport scientists.

Many athletes believe supplementation with multi-ingredient performance supplement (MIPS) prior to training will result in greater focus, shorter reaction time, and increased power $(4,5)$. Therefore, the beneficial properties of such products for team sport athletes may not be effective in increasing endurance or/and strength performance per se but rather in improving decision-making and perceptual skills. As was proven in the studies by Kaczka et al. (6) MIPS significantly improves upper and lower body strength in isokinetic test and power output measured in Wingate Anaerobic Test in resistance trained men. The most popular and examined ingredient of pre-workout formulations is caffeine which enhances performance through peripheral and central mechanisms (7). Research indicate that decision-making and perceptual skills may be improved by caffeine ingestion however results remain unclear. Lorino et al. (8) found no improvement in total time (TT) to complete a proagility run following ingestion of 6 $\mathrm{mg} / \mathrm{kg}$ body mass $(\mathrm{BM})$ of caffeine (1 h before exercise). In contrast, Stuart et al. (9) reported 
that caffeine ingestion $(6 \mathrm{mg} / \mathrm{kg} \mathrm{BM})$ resulted in a 2,2\% improvement in mean performance across three separate reactive agility sprints in zig-zag manner. It is important that both of these studies used preplanned movement tests, which do not require to "read and react" - thus perceptual component of reactive agility has been eliminated. To the current knowledge of authors only one study showed improved reactive agility in team-sport athletes after $6 \mathrm{mg} / \mathrm{kg}$ caffeine ingestion 60 min prior to exercise (10).

It is believed that ingredients found in many MIPS such as caffeine, beta-alanine, citrulline and plant ingredients which target different physiological mechanisms may elicit synergistic effect and in turn improve athletic performance $(4 ; 11)$. For example hordenine, a constituent of barley and beer, has approximately identical ligand efficacy as dopamine (76\%) (12). Hordenine is also found in Citrus aurantium which has been shown to have influence on adrenergic receptors by stimulating the release of noradrenaline (norepinephrine $-\mathrm{NE})(13,14)$. Therefore, it could be hypothesized that with caffeine and other sympathomimetic ingredients of different MIPS could have greater potentiating effect on performance. Moreover, dopaminergic and catecholaminergic effect may be also enhanced by tyrosine, which is found in many pre-workouts. Norepinephrine, along with dopamine, has come to be recognized to play significant role in focus and alertness. This is perhaps not surprising given the location and distribution of NE neurons throughout the brain (15). In study by Spradley et al. (16) choice reaction time was improved by consuming MIPS containing: BCAAs, creatine, beta-alanine, citrulline malate, arginine, vitamin B6, vitamin B12, and caffeine. Although it is unknown if improvement was due to caffeine or because of synergistic effect of other ingredients. Multiple ingredients potentially interact and these interactions may potentiate or attenuate supplement effectiveness. Pre-workout supplements typically consist of multiple active ingredients, which once ingested, can modify pharmacodynamics and pharmacokinetics resulting in different bioavailability properties and physiological effects. Moreover most of the research regarding 
effectiveness of pre-training supplements focused on fatigue delay or/and improving quality of resistance training however its effects on reactive agility is lacking and needs further research $(4,12,16,17,18,19)$

Therefore, the purpose of this investigation was to examine the acute effects of a commercially available multi-ingredient pre-workout performance supplement in comparison to caffeine, on reactive agility and on jump height, in recreationally-trained handball male players. It was hypothesized that the supplement would improve reactive agility decisionmaking skills relative to placebo and caffeine due to the presence of biologically-active ingredients, that may enhance cognitive performance, as well as preventing the decrease in lower limbs power after reactive agility tests. The results of these studies may contribute to the assessment of the usefulness of MIPS type supplementation in comparison to the supplementation of equal amount of caffeine alone in enhancing reactive agility and jump performance

\section{Methods}

\section{Study design}

Following an explanation of all procedures, risks, and benefits associated with the study, each subject gave his written consent before he participated in the study. The study was approved by the Ethical Committee of the Academy of Physical Education in Katowice (Katowice, Poland; Resolution No. 2/2018) and conformed to the ethical requirements of the 1975 Helsinki Declaration. The study followed a randomized double-blind, crossover design. All subjects attended a familiarization session one week before testing. During the familiarization session the participants were shown and explained the planned tests as well as their order on one day and their order during the entire experiment. They were also instructed not to consume caffeine containing products 24 hours before testing, to reduce the effect of caffeine tolerance. The subjects were asked to refrain from heavy exercise and alcohol 
consumption during period of the experiment. In addition, subjects were instructed not to eat or drink for three hours prior to each trial. Subjects underwent reactive agility test on three separate days with $72 \mathrm{~h}$ apart between test days. Just after accomplishing of each repetition of reactive agility tests participants were asked to perform countermovement jump (CMJ; two repetitions separated by one minute break) to establish the average height of jump, that is directly related to lower body power.

After consumption of either PL or the supplement solution, subjects took a 15 minutes rest prior to commencing the warm-up. The standard warm-up started with 10 min jogging at $60 \%-75 \%$ of maximal heart rate. After this task, the participants performed various dynamic exercises for five minutes (arm swing, internal/external leg rotation, hip flexion/extension/ abduction/adduction/ hip rotation, knee rotation, and ankle rotation) followed by $15 \mathrm{~min}$ jogging.

\section{Supplementation}

Forty-five minutes before testing subjects were randomly provided with either placebo $(\mathrm{PL})-250 \mathrm{ml}$ of the flavored water; caffeine (CAF) - flavored water containing two ingredients: anhydrous caffeine (200 mg) and guarana extract (200 mg) - $300 \mathrm{mg}$ of caffeine in total, mixed with water $(250 \mathrm{ml})$, or MIPS mixed with $250 \mathrm{ml}$ of water. The MIPS supplement which is commercially marketed as Knockout $2.0^{\circledR}$ (Olimp Laboratories, Pustynia, Poland) consisted of 9.6 g powder mixed with water $(250 \mathrm{ml})$. The powder contained: L-citrulline $(3 \mathrm{~g})$, beta-alanine (2 g), taurine (750 mg), L-arginine (500 mg), L - tyrosine, anhydrous caffeine (200 mg), guarana extract (200 mg; in total $300 \mathrm{mg}$ of caffeine), barley - derived hordenine extract (150 mg), cayenne pepper seed extract $(25 \mathrm{mg})$, black pepper extract $(7.5 \mathrm{mg})$ and Huperzia serrata extract $(3 \mathrm{mg})$.

It is need to be emphasize that the amount and chemical form of caffeine consumed by subjects was the same in the caffeine (CAF) and MIPS groups and its dosage was $5 \mathrm{mg} / \mathrm{kg}$. Anhydrous 
caffeine was provided by Biesterfeld International Polska Sp z o.o., Warsaw, Poland) and guarana extract (standardized for 50\% caffeine content) was provided by EVER Pharma, Lyon, France).

\section{Participants}

Twenty-four recreationally-trained handball male players (body mass $=74.6 \pm 8.8[\mathrm{~kg}]$; height $=179 \pm 7.2[\mathrm{~cm}]$; age $=23.8 \pm 1.4$ years $)$ underwent three testing sessions conducted in a randomized and double-blind fashion manner. Following the explanation of all procedures, risks, and benefits associated with the study, each subject gave his written consent prior to participation. Subjects were also required to have been free of any nutritional supplements or ergogenic aids use for the two weeks preceding the study. They were also asked to refrain from taking any additional supplement and coffee or strong tee in order to exclude potential sources of caffeine during the time of the study.

\section{Reactive agility test- Test 1-1-2 (Y-test)}

Four pairs of electronic, single-beamed, infrared timing gates (Fusion Smart Speed PRO, Brisbane, Australia) were positioned as shown in Figure 1. The timing gate system dictated the direction in which participants proceeded having completed the first $5 \mathrm{~m}$ of the course. Participants began each trial $20 \mathrm{~cm}$ behind the starting gates before running at maximum speed in a straight line toward second timing gate. Upon breaking the beam of the timing gate in the middle of the course (abort gates), lights on either the left or right exit gate flashed. Participants were required to react to this stimulus and sprint as quickly as possible the next 10 $\mathrm{m}$, through the illuminated timing gate. Sprint times were recorded telemetrically, with all data transmitted to a Personal Digital Assistant. During the reactive agility sprints, participants were instructed not to try to predict which exit gate they would be required to sprint through; to ensure that this did not occur, the investigator visually monitored technique and compared reactive performance times to planned sprint times. Participants had completed the test until 

test 1-1-2 scheme is shown in Figure 1.

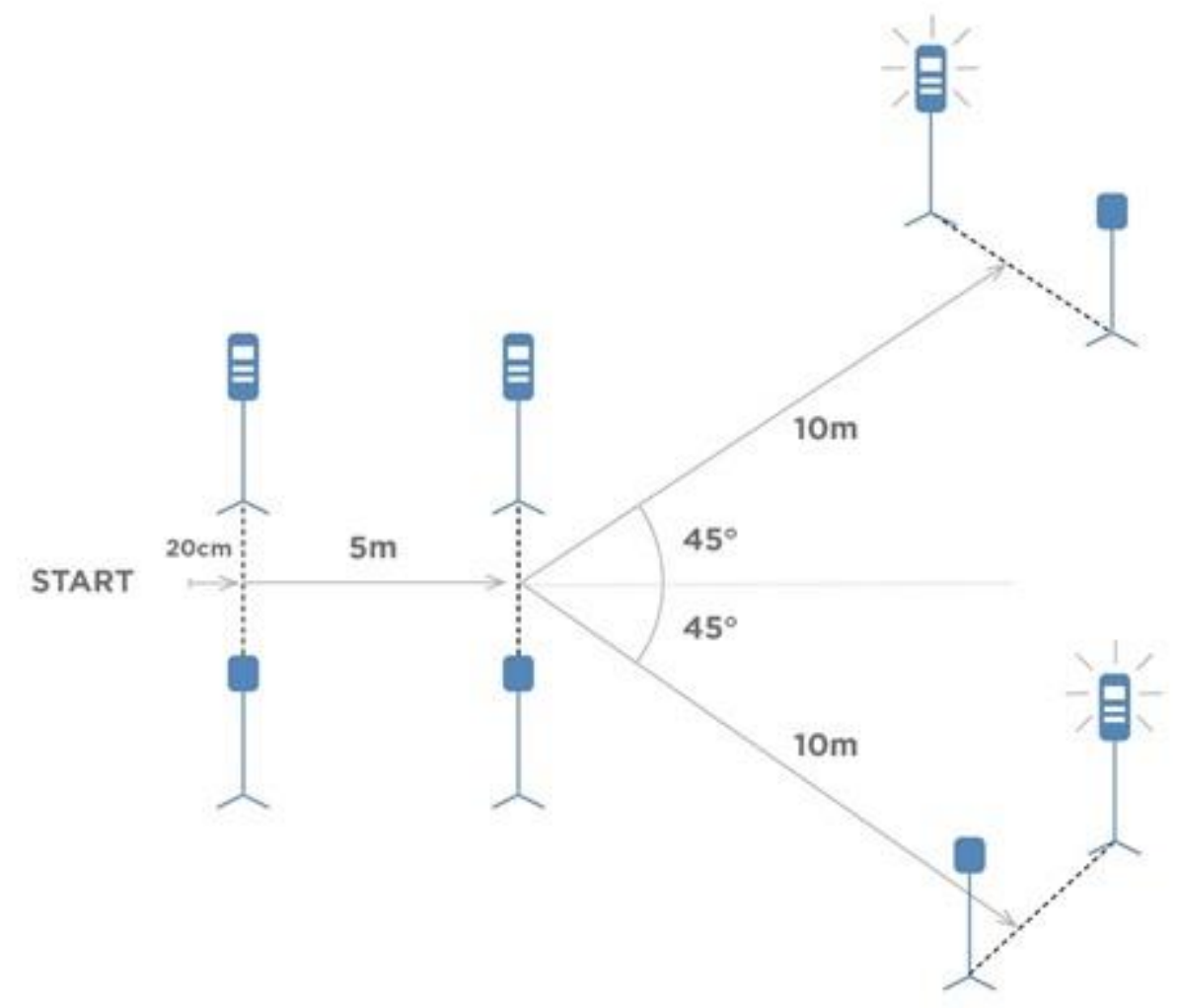

171 Figure 1. The layout of photocell timing gates and the way of carrying out the test task

172 protocol 1-1-2.

173

\section{Countermovement jump}

The countermovement jump, subjects started in a standing position between Optojump system (Optojump, Microgate, Bolzano, Italy) consists of two bars (transmitting and receiving bars, $1 \mathrm{~m}$ apart) equipped with 33 optical LEDs fitted in the transmitting bar that continuously communicate with the corresponding set in the receiving bar. The LEDs are positioned $0.3 \mathrm{~cm}$ from ground level. Any break of the beam switched on and off automatically activates a digital chronometer used to calculate Flight Time and Jump Height with feet shoulder width apart. Subject performed an explosive jump using a slow stretch-shortening cycle (SSC) at $\sim 90^{\circ}$ knee flexion. Subjects were asked to jump at maximal effort and as high as possible. The modality 
of the CMJ included the arm swing during the execution of the movement and hands were free to move. At every session all subjects performed two trials for each jump test, with a $60 \mathrm{~s}$ interval between each trial. The better result of these two trials was taken for further analysis $(20,21,22)$.

\section{Statistical analysis}

The consistency of distribution regarding the assessed indices with normal distribution was investigated with the Shapiro-Wilk test. To assess the significance of differences between the groups studied, Friedmann ANOVA test was used, the chi-square value $\left(\chi^{2}\right)$ calculated and the $p$ value of statistical significance estimated. In the case of $p<0.05$, inter-group comparisons using Wilcoxon test were made. Data presented as mean and standard deviation $( \pm$ SD). All calculations were carried out in the program Statistica (StatSoft, USA).

\section{Results}

The research indicated significant inter-group differences in reactive agility test $(\mathrm{p}=0.04)$. Time needed to complete the $1-1-2$ test was significantly shorter for MIPS supplementation in comparison to PL $(-3.4 \% ; \mathrm{p}=0.02)$ and to CAF $(-2.8 \% ; \mathrm{p}=0.004)$. But not significantly important between PL and CAF groups $(\mathrm{p}=0.6)$

CMJ results are presented in Table 1 and Figure 2. We have observed significant improvements ( $\mathrm{p}=0.001)$ in jump height. For MIPS group in comparison to PL the jump was higher $(+5.2 \% ; \mathrm{p}=0.001)$ and $\mathrm{CAF}(+5.1 \% ; \mathrm{p}=0.001)$ groups, but not $\mathrm{CAF}$ to PL group $(\mathrm{p}=0.84)$.

Table 1. Effects of supplementation on reactive agility test and countermovement jump (PL placebo, MIPS- multi-ingredient performance supplement, and CAF- caffeine group) 


\begin{tabular}{|c|c|c|c|c|c|}
\hline TEST & PL & MIPS & CAF & $\begin{array}{c}\text { ANOVA } \\
\text { p } \\
\left(\chi^{2}\right)\end{array}$ & $\begin{array}{c}\text { Wilcoxon test } \\
\text { p }\end{array}$ \\
\hline $\begin{array}{c}\text { 1-1-2 } \\
{[\mathrm{s}]}\end{array}$ & $\begin{array}{r}2.595 \\
\pm 0.142\end{array}$ & $\begin{array}{r}2.507 \\
\pm 0.087\end{array}$ & $\begin{array}{r}2.580 \\
\pm 0.102\end{array}$ & $\begin{array}{l}0.04 \\
(6.33)\end{array}$ & $\begin{array}{c}\text { PL-MIPS: } 0.02 \\
\text { CAF-MIPS: } 0.004 \\
\text { PL-CAF: } 0.6\end{array}$ \\
\hline $\begin{array}{l}\text { CMJ } \\
{[\mathrm{cm}]}\end{array}$ & $\begin{array}{r}48.12 \\
\pm 4.09\end{array}$ & $\begin{array}{r}50.61 \\
\pm 4.01\end{array}$ & $\begin{array}{l}48.16 \\
\pm 4.19\end{array}$ & $\begin{array}{c}0.001 \\
(33.58)\end{array}$ & $\begin{array}{c}\text { PL-MIPS: } 0.001 \\
\text { CAF-MIPS: } 0.001 \\
\text { PL-CAF: } 0.84\end{array}$ \\
\hline
\end{tabular}

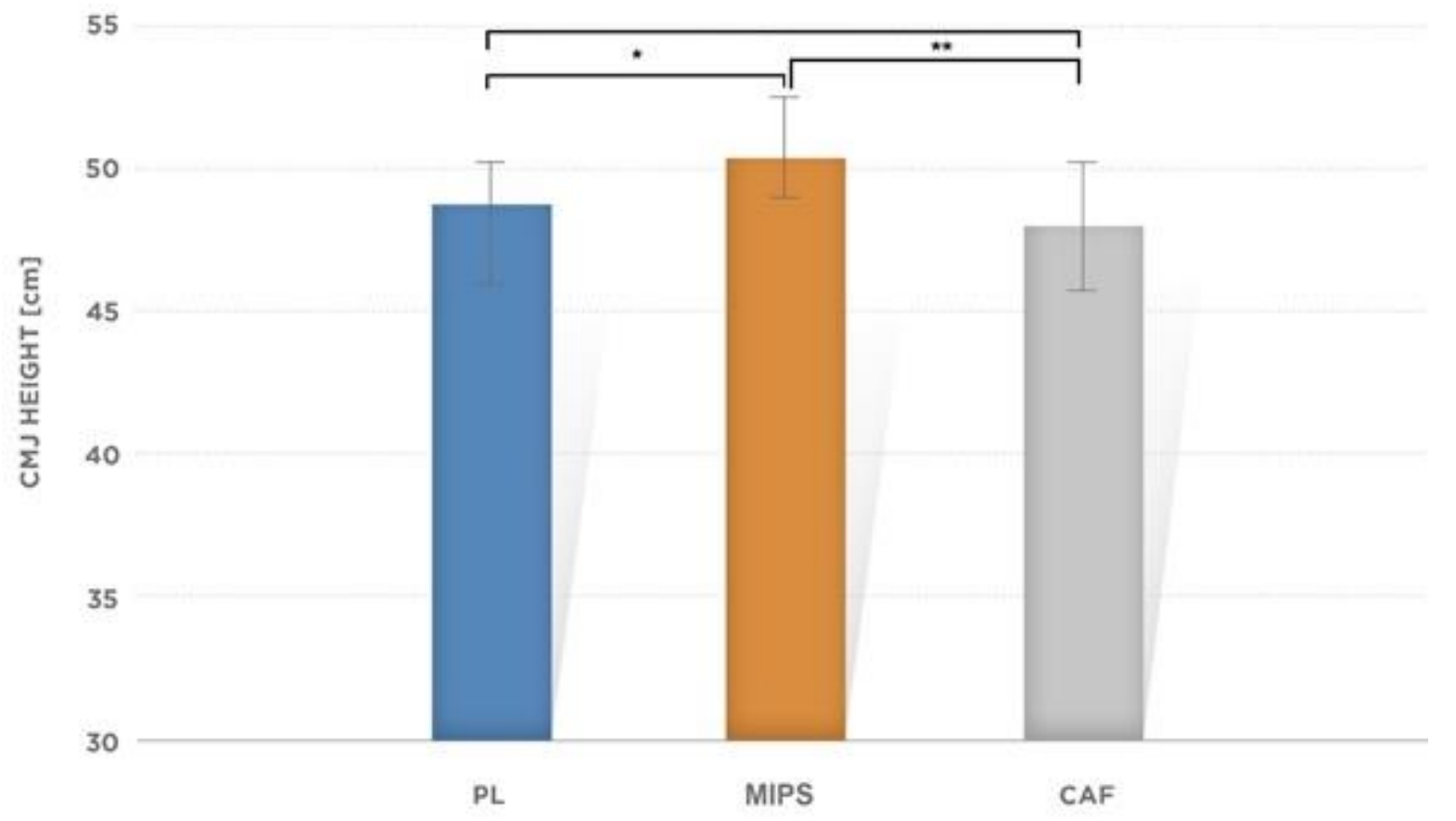

207 Figure 2. The mean values of countermovement jump (cm) after PL, MIPS or CAL ingestion.

208 Significant difference in jump height was observed between: MIPS and PL groups $\left({ }^{*} \mathrm{p}=0.001\right)$

209 as well as between MIPS and CAF groups (** $\mathrm{p}=0.001)$, but not CAF and PL groups ( $\mathrm{p}=0.84)$.

210 Error bars indicate standard deviation (SD). 


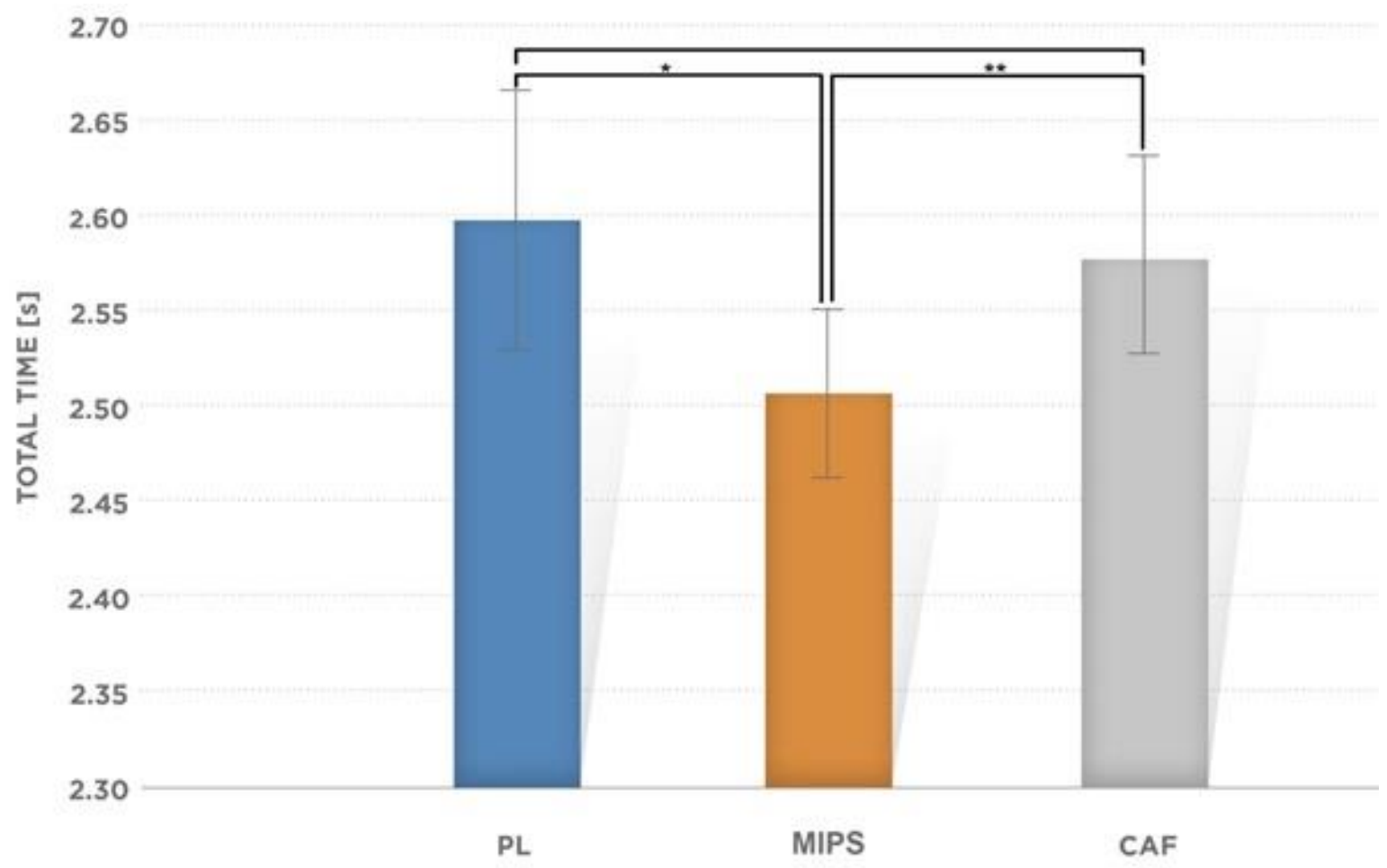

Figure 3. The mean values of total time (s) as result of 1-1-2 reactive agility test protocol after PL, MIPS or CAF ingestion. Significant difference in total time was observed between: MIPS and PL groups $(* p=0.02)$, as well as MIPS and CAF groups $(* * p=0.004)$, but not CAF and PL groups $(\mathrm{p}=0.06)$. Error bars indicate standard deviation $(\mathrm{SD})$.

\section{Discussion}

The results of this study indicate that multi-ingredient performance supplement can significantly improve reactive agility, and increase jump height. As was proven in the studies by Kaczka et al. (6) exactly the same supplement significantly improves upper and lower body strength (isokinetic test) and power output in Wingate Anaerobic test in resistance trained men. 
At the same time, acute ingestion of this supplement had significant and beneficial effect on anaerobic power. This is the first study comparing MIPS and the same amount as well as chemical form of caffeine on reactive agility. Caffeine can influence central nervous system and enhance the cognitive functions like arousal and the ability to concentrate (23). It is well established that caffeine in doses from 32 to $300 \mathrm{mg}$ (or roughly $0.5-4.0 \mathrm{mg} / \mathrm{kg} \mathrm{BM}$ for a $75 \mathrm{~kg}$ individual) enhances fundamental aspects of cognitive performance, such as attention, vigilance, and reaction time $(24,25,26)$. However, the limitation of current studies was that none of them tested the effect of caffeine on reactive agility in a game setting, i.e. in combination with a perceptual component that requires the participant to initiate the movement response as opposed to such pre-planned tests (3). It should be noted, there is general consensus that caffeine improves "lower" cognitive functions such as simple reaction time, whereas caffeine's effects on "higher" cognitive functions such as problem solving and decision making are often debated (27). We did not show any improvement in the CAF group in the 1-1-2 run test in comparison to placebo. The dose used $(5 \mathrm{mg} / \mathrm{kg})$ might have been too low for the fitness level of the study group. Pontifex et al. (28) indicated, that even higher doses of caffeine (6 $\mathrm{mg} / \mathrm{kg} \mathrm{BM}$ ) had no influence on reactive agility time measured with modality similar to the one in this study.

Only study by Duvnjak - Zaknich et al. (10) assessed agility, requiring participants to react appropriately to a sport-specific video stimulus after caffeine ingestion. Authors, in contrast to our results, observed improvement in decision-making and movement time before and after four, 20 min circuits that replicated the movement patterns and exercise demands in team sports. Discrepancy across these findings can be attributed to the type of the stimulus (light vs. video-opponent) and caffeine dose which was almost two times higher in Duvnjak Zaknich et al. (10) study. How caffeine dose affects performance depends in part on the arousal level of the individuals under investigation, especially the extent to which subjects are sleep- 
deprived or fatigued versus well-rested $(25,29)$. Giving the same dose to someone who already is well-rested and highly aroused may degrade rather than improve performance because in this case, caffeine produces a state of over-arousal, which according to the Yerkes-Dodson law, will degrade cognition $(29,30,31)$. Research has shown that better performance can be distinguished from lower skilled athletes by the ability to quickly and accurately react to opponent's movements, but not to a generic stimulus such as a flashing light. Despite the importance of maintaining alertness and attentional focus in team games athletes, there exists a lack of research investigating sport-specific reaction time and this prevents any firm conclusion being drawn on the ability of caffeine to improve the reactive agility. Whereas, ingestion of the pre-workout significantly improved reactive agility compared to both placebo and caffeine conditions. Because we have not examined the effect of the combination of all individual ingredients of MIPS with caffeine and their effect alone on the exercise performance outcomes, we are unable to identify which ingredient could be the most responsible for the potentially synergistic effect. We can suggest that effect of caffeine could be enhanced by other ingredients which share similar mechanism of central nervous system stimulation. For example pepperderived alkaloids such as capsaicin and piperine are found to have thermogenic and bioenergetic effects which are triggered by activation of thermoreceptors and release of catecholamines (32). Guarana seeds in addition to caffeine, contains a number of other possible stimulants such as flavonoids, or other potentially psychoactive components, including saponins and tannins, which could enhance cognitive function $(33,34)$.

Relatively high values of CMJ height, presented in this study, may be due to the used modality, that included arm swing and the depth of the countermovement directly before the jump (20). As other authors stated $-5 \mathrm{~cm}$ increase in countermovement depth significantly influences jump performance (35). Higher jump and joint angular velocities were also observed wen the knee flexion range increased from $70^{\circ}$ to $90^{\circ}(36)$. 
There are many studies $(37,38)$ concentrating on the effect of caffeine on endurance exercise but the influence of oral administration of this substance on jump performance remains unclear $(7,39)$. The research of Bloms et al. (40) indicates $4.1 \%$ increase in CMJ height after the ingestion of $5 \mathrm{mg} / \mathrm{kg} \mathrm{BM}$ of caffeine in young males (18-23 years) recruited from the National Collegiate Athletic Division. It is worth noting that all of the subjects trained disciplines involving maximal-intensity ballistic tasks. The authors stated that the effect of ergogenic aid, such as oral caffeine administration, is more apparent in highly trained athletes. Moreover the effect tends to be stronger for exercises involving large muscle groups (41). Another study conducted in the group of elite volleyball players also showed improvements in CMJ parameters like: flight time $(+5.3 \%)$, peak power $(+16.2 \%)$ and peak concentric force $(+6.5 \%)$ without any side effects of caffeine ingestion in the amount of $5 \mathrm{mg} / \mathrm{kg}(42)$. Protocol used in this research did not show any changes in the CMJ height after caffeine ingestion. That might be due to the group involved - young, recreationally-trained handball male players students but not experienced athletes, for which the range of the results would probably be more stable. It can also be postulated, that the significant difference between PL and MIPS group is due to the synergistic effect of caffeine and other constituents of the pre-workout ingredients $(6,19)$. There is very limited data on the effect of acute MIPS ingestion and its effect on lower limbs power measured by CMJ or vertical jump. It is not possible to compare some of the research with this experiment. Unlike the supplement in this study, MIPS used in mentioned publications often do not clearly declare the amount of each ingredient, what is due to different labeling system.

\section{Limitations of the study}

Given the scarcity of research on the MIPS blend composition, more research is warranted to gain a better understanding of their effects on sport performance and reactive agility, the more that the presence of additional biologically-active ingredients in the multi- 
ingredient performance supplement may act synergistically with caffeine and thereby enhance cognitive performance, as well as preventing lower limbs power after reactive agility tests. The assessment of the effect of individual components of the blend composition of the MIPS in combination with caffeine seems to be crucial in understanding which of them is the most effective at the doses tested.

\section{Conclusion}

The results of this study indicate that pre-exercise ingestion of the MIPS-type product, can significantly improve reactive agility performance, and jump height to the grater extend than the same amounts of caffeine alone consumed at the same time or consuming placebo.

\section{Declarations}

\section{Abbreviations}

MIPS: multi-ingredient performance supplement; PL: placebo group; MIPS: multi-ingredient performance supplement supplemented group; CAF: caffeine group SD: standard deviations; CMJ: countermovement jump; TT: total time; BM: body mass; NE: norepinephrine; BCAAs: branched-chain amino acids; Y-test or 1-1-2 test: reactive agility test 1-1-2; BW: body weight.

Ethics approval and consent to participate: The study was approved by the Ethical Committee of the University School of Physical Education in Katowice (Katowice, Poland; Resolution No. 2/2018) and conformed to the ethical requirements of the 1975 Helsinki Declaration. All participants were informed about risk and benefits associated with the study and provided voluntary, written, informed consent.

Authors' contributions: Conceptualization: $\mathrm{PK}, \mathrm{AB}$ and $\mathrm{KK}$; methodology: $\mathrm{PK}, \mathrm{AB}$ and $\mathrm{KK}$; investigation and data collection: $\mathrm{PK}, \mathrm{AB}, \mathrm{RJ}, \mathrm{HZ}, \mathrm{TZ}$; analysis and interpretation: $\mathrm{PK}, \mathrm{AB}$, KK, EK, JB, HZ and MM; statistical analysis: MM; writing, original draft preparation: PK, 
KK; writing and editing: $\mathrm{PK}, \mathrm{AB}, \mathrm{KK}, \mathrm{MM}, \mathrm{EK}, \mathrm{JB}$, TZ; supervision: PK. All authors have read and agreed to the published version of the manuscript.

Acknowledgments: Not applicable

Consent for publication: Not applicable

Availability of data and materials: The datasets used and/or analyzed during the current study are available from the corresponding author on reasonable request.

Competing interests: The authors declare that they have no competing interests.

Funding: This research received no external funding.

\section{Figures:}

Figure 1. The layout of photocell timing gates and the way of carrying out the test task protocol 1-1-2.

Figure 2. The mean values of countermovement jump (cm) after PL, MIPS or CAL ingestion. Significant difference in jump height was observed between: MIPS and PL groups $\left({ }^{*} \mathrm{p}=0.001\right)$ as well as between MIPS and CAF groups (**p=0.001), but not CAF and PL groups ( $\mathrm{p}=0.84)$. Error bars indicate standard deviation (SD).

Figure 3. The mean values of total time (s) as result of 1-1-2 reactive agility test protocol after PL, MIPS or CAF ingestion. Significant difference in total time was observed between: MIPS and PL groups $(* \mathrm{p}=0.02)$, as well as MIPS and CAF groups $(* * \mathrm{p}=0.004)$, but not CAF and PL groups $(\mathrm{p}=0.06)$. Error bars indicate standard deviation (SD).

\section{References}


1 Sheppard JM, Young WB. Agility literature review: classifications, training and testing. J Sports Sci. 2006 Sep;24(9):919-32.

2 Nimphius S, Callaghan SJ, Bezodis NE, Lockie RG. Change of Direction and Agility Tests: Challenging Our Current Measures of Performance. Strength Cond J. 2017 Aug 1;40(1):1.

3 Chia JS, Barrett LA, Chow JY, Burns SF. Effects of Caffeine Supplementation on Performance in Ball Games. Sports Med. 2017 Dec;47(12):2453-71.

4 Jagim AR, Jones MT, Wright GA, St Antoine C, Kovacs A, Oliver JM. The acute effects of multi-ingredient pre-workout ingestion on strength performance, lower body power, and anaerobic capacity. J Int Soc Sports Nutr. 2016 Mar 8;13:11.

5 Froiland K, Koszewski W, Hingst J, Kopecky L. Nutritional supplement use among college athletes and their sources of information. Int J Sport Nutr Exerc Metab. 2004 Feb;14(1):104-20.

6 Kaczka P, Batra A, Kubicka K, Maciejczyk M, Rzeszutko-Bełzowska A, Pezdan-Śliż I, et al. Effects of PreWorkout Multi-Ingredient Supplement on Anaerobic Performance: Randomized Double-Blind Crossover Study. Int J Environ Res Public Health. 2020 Nov 9;17(21).

7 Davis JK, Green JM. Caffeine and anaerobic performance: ergogenic value and mechanisms of action. Sports Med. 2009;39(10):813-32.

8 Lorino AJ, Lloyd LK, Crixell SH, Walker JL. The effects of caffeine on athletic agility. J Strength Cond Res. 2006;20(4):851-4

9 Stuart GR, Hopkins WG, Cook C, Cairns SP. Multiple effects of caffeine on simulated high-intensity team-sport performance. Med Sci Sports Exerc. 2005 Nov;37(11):1998-2005.

10 Duvnjak-Zaknich DM, Dawson BT, Wallman KE, Henry G. Effect of caffeine on reactive agility time when fresh and fatigued. Med Sci Sports Exerc. 2011 Aug;43(8):1523-30.

11 Gonzalez AM, Walsh AL, Ratamess NA, Kang J, Hoffman JR. Effect of a pre-workout energy supplement on acute multi-joint resistance exercise. J Sports Sci Med. 2011 Jun 1;10(2):261-6.

12 Sommer T, Hübner H, El Kerdawy A, Gmeiner P, Pischetsrieder M, Clark T. Identification of the Beer Component Hordenine as Food-Derived Dopamine D2 Receptor Agonist by Virtual Screening a 3D Compound Database. Sci Rep. 2017 Mar 10;7:44201.

13 Barwell CJ, Basma AN, Lafi MA, Leake LD. Deamination of hordenine by monoamine oxidase and its action on vasa deferentia of the rat. J Pharm Pharmacol. 1989 Jun;41(6):421-3.

14 Slezak T, Francis PS, Anastos N, Barnett NW. Determination of synephrine in weight-loss products using high performance liquid chromatography with acidic potassium permanganate chemiluminescence detection. Anal Chim Acta. 2007 Jun 12;593(1):98-102.

15 Tyrosine Supplementation: Can This Amino Acid Boost Brain Dopamine and Improve Physical and Mental Performance? [Internet]. [cited 2020 Nov 19]. Available from: https://www.gssiweb.org/sports-scienceexchange/article/sse-157-tyrosine-supplementation-can-this-amino-acid-boost-brain-dopamine-and-improvephysical-and-mental-performance

16 Spradley BD, Crowley KR, Tai C-Y, Kendall KL, Fukuda DH, Esposito EN, et al. Ingesting a pre-workout supplement containing caffeine, B-vitamins, amino acids, creatine, and beta-alanine before exercise delays fatigue while improving reaction time and muscular endurance. Nutr Metab. 2012 Mar 30;9:28.

17 Hoffman JR, Kang J, Ratamess NA, Jennings PF, Mangine GT, Faigenbaum AD. Effect of nutritionally enriched coffee consumption on aerobic and anaerobic exercise performance. J Strength Cond Res. 2007 May;21(2):456-9.

18 Hoffman JR, Ratamess NA, Ross R, Shanklin M, Kang J, Faigenbaum AD. Effect of a pre-exercise energy supplement on the acute hormonal response to resistance exercise. J Strength Cond Res. 2008 May;22(3):874-82.

19 Martinez N, Campbell B, Franek M, Buchanan L, Colquhoun R. The effect of acute pre-workout supplementation on power and strength performance. J Int Soc Sports Nutr. 2016 Jul 16;13:29.

20 Attia A, Dhahbi W, Chaouachi A, Padulo J, Wong DP, Chamari K. Measurement errors when estimating the vertical jump height with flight time using photocell devices: the example of Optojump. Biol Sport. 2017 Mar;34(1):63-70.

21 Claudino JG, Cronin J, Mezêncio B, McMaster DT, McGuigan M, Tricoli V, et al. The countermovement jump to monitor neuromuscular status: A meta-analysis. J Sci Med Sport. 2017 Apr;20(4):397-402.

22 Petrigna L, Karsten B, Marcolin G, Paoli A, D'Antona G, Palma A, et al. A Review of Countermovement and Squat Jump Testing Methods in the Context of Public Health Examination in Adolescence: Reliability and Feasibility of Current Testing Procedures. Front Physiol. 2019 Nov 7;10:1384.

23 Goldstein ER, Ziegenfuss T, Kalman D, Kreider R, Campbell B, Wilborn C, et al. International society of sports nutrition position stand: caffeine and performance. J Int Soc Sports Nutr. 2010 Jan 27;7(1):5.

24 Lorist MM, Snel J. Caffeine, Sleep, and Quality of Life. In: Verster JC, Pandi-Perumal SR, Streiner DL, editors. Sleep and Quality of Life in Clinical Medicine. Totowa, NJ: Humana Press; 2008. p. 325-32. 25 Nehlig A. Is caffeine a cognitive enhancer? J Alzheimers Dis. 2010;20 Suppl 1:S85-94. 
26 Snel J, Lorist MM, Tieges Z. Coffee, caffeine, and cognitive performance. In: Coffee, Tea, Chocolate and the Brain. CRC Press; 2004. p. 53-71..

27 Kosslyn MM, Smith EE. Higher cognitive functions - introduction. In: Gassaniga MS (Ed.), The New Cognitive Neurosciences, The MIT Press, Cambridge, U.K (2001), pp. 961-964

28 Pontifex KJ, Wallman KE, Dawson BT, Goodman C. Effects of caffeine on repeated sprint ability, reactive agility time, sleep and next day performance. J Sports Med Phys Fitness. 2010 Dec;50(4):455-64.

29 McLellan TM, Caldwell JA, Lieberman HR. A review of caffeine's effects on cognitive, physical and occupational performance. A review of caffeine's effects on cognitive, physical and occupational performance

30 Yerkes RM, Dodson JD. The Relation of Strength of Stimulus to Rapidity of Habit Formation. Journal of Comparative Neurology \& Psychology. 1908;18:459-82.

31 Wood S, Sage JR, Shuman T, Anagnostaras SG. Psychostimulants and cognition: a continuum of behavioral and cognitive activation. Pharmacol Rev. 2014;66(1):193-221.

32 Dudhatra GB, Mody SK, Awale MM, Patel HB, Modi CM, Kumar A, et al. A comprehensive review on pharmacotherapeutics of herbal bioenhancers. ScientificWorldJournal. 2012 Sep 17;2012:637953.

33 Hamerski L, Somner GV, Tamaio N. Paullinia cupana Kunth (Sapindaceae): A review of its ethnopharmacology, phytochemistry and pharmacology. Available from: http://dx.doi.org/10.5897/JMPR2013.5067

34 Pomportes L, Brisswalter J, Casini L, Hays A, Davranche K. Cognitive Performance Enhancement Induced by Caffeine, Carbohydrate and Guarana Mouth Rinsing during Submaximal Exercise. Nutrients [Internet]. 2017 Jun 9;9(6). Available from: http://dx.doi.org/10.3390/nu9060589

35 Sánchez-Sixto A, Harrison AJ, Floría P. Larger Countermovement Increases the Jump Height of Countermovement Jump. Sports (Basel) [Internet]. 2018 Oct 26;6(4). Available from: http://dx.doi.org/10.3390/sports6040131

36 Moran KA, Wallace ES. Eccentric loading and range of knee joint motion effects on performance enhancement in vertical jumping. Hum Mov Sci. 2007 Dec;26(6):824-40.

37 Graham TE, Spriet LL. Metabolic, catecholamine, and exercise performance responses to various doses of caffeine. J Appl Physiol. 1995 Mar;78(3):867-74.

38 Graham TE, Hibbert E, Sathasivam P. Metabolic and exercise endurance effects of coffee and caffeine ingestion. J Appl Physiol. 1998 Sep;85(3):883-9.

39 Astorino TA, Roberson DW. Efficacy of acute caffeine ingestion for short-term high-intensity exercise performance: a systematic review. J Strength Cond Res. 2010 Jan;24(1):257-65.

40 Bloms LP, Fitzgerald JS, Short MW, Whitehead JR. The Effects of Caffeine on Vertical Jump Height and Execution in Collegiate Athletes. J Strength Cond Res. 2016 Jul;30(7):1855-61.

41 Timmins TD, Saunders DH. Effect of caffeine ingestion on maximal voluntary contraction strength in upperand lower-body muscle groups. J Strength Cond Res. 2014 Nov;28(11):3239-44.

42 Zbinden-Foncea H, Rada I, Gomez J, Kokaly M, Stellingwerff T, Deldicque L, et al. Effects of Caffeine on Countermovement-Jump Performance Variables in Elite Male Volleyball Players. Int J Sports Physiol Perform. 2018 Feb 1;13(2):145-50. 
Figures

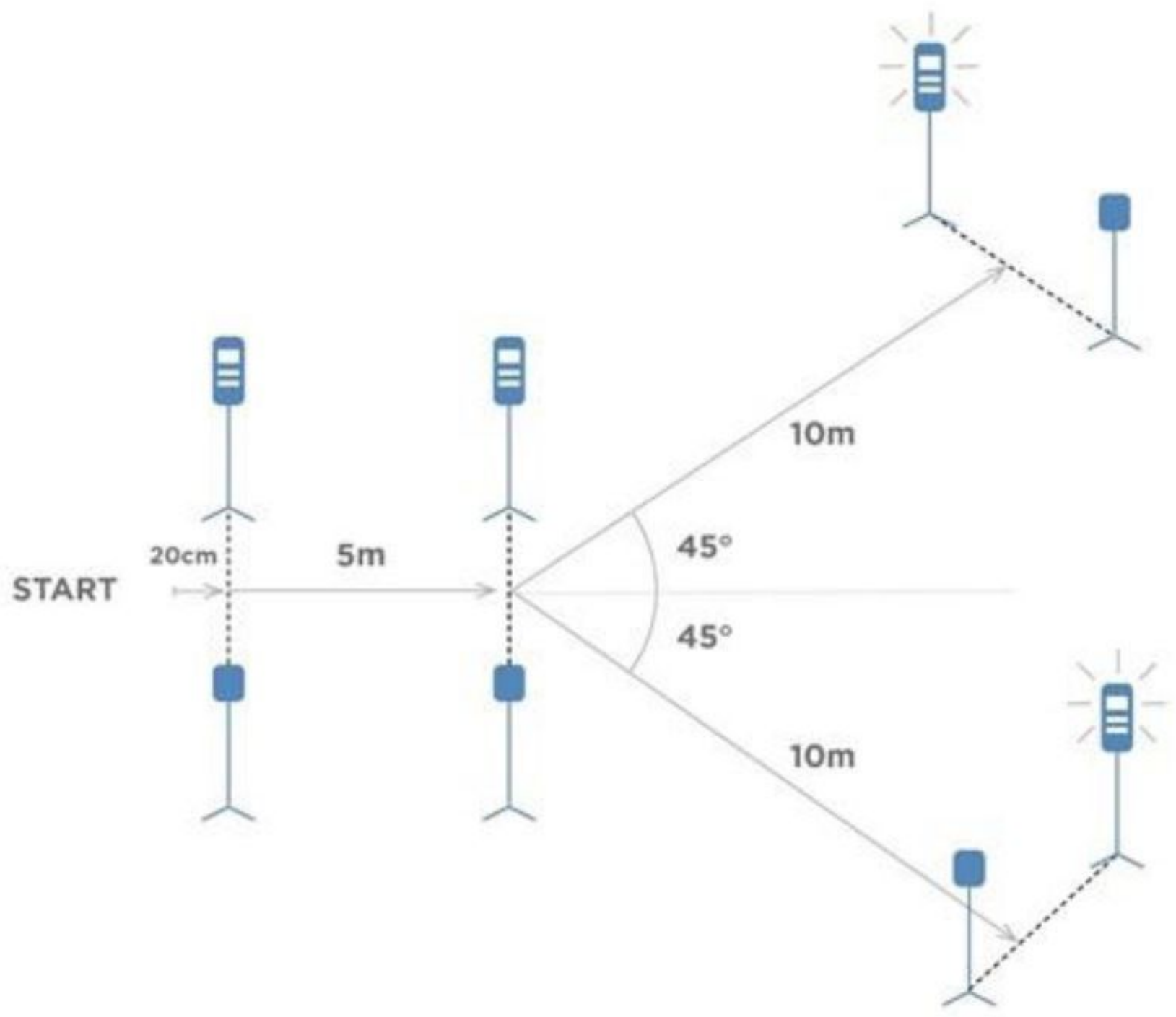

\section{Figure 1}

The layout of photocell timing gates and the way of carrying out the test task protocol 1-1-2. 


\begin{tabular}{|c|c|c|c|c|c|}
\hline TEST & PL & MIPS & CAF & $\begin{array}{c}\text { ANOVA } \\
\left(\chi^{2}\right)\end{array}$ & $\begin{array}{c}\text { Wilcoxon test } \\
\text { p }\end{array}$ \\
\hline $\mathbf{1 - 1 - 2}$ & 2.595 & 2.507 & 2.580 & 0.04 & PL-MIPS: 0.02 \\
{$[\mathbf{s}]$} & \pm 0.142 & \pm 0.087 & \pm 0.102 & $(6.33)$ & CAF-MIPS: 0.004 \\
\hline $\mathbf{C M J}$ & 48.12 & 50.61 & 48.16 & 0.001 & PL-CAF: 0.6 \\
[cm] & \pm 4.09 & \pm 4.01 & \pm 4.19 & $(33.58)$ & CAF-MIPS: 0.001 \\
& & & & & PL-CAF: 0.84 \\
\hline
\end{tabular}

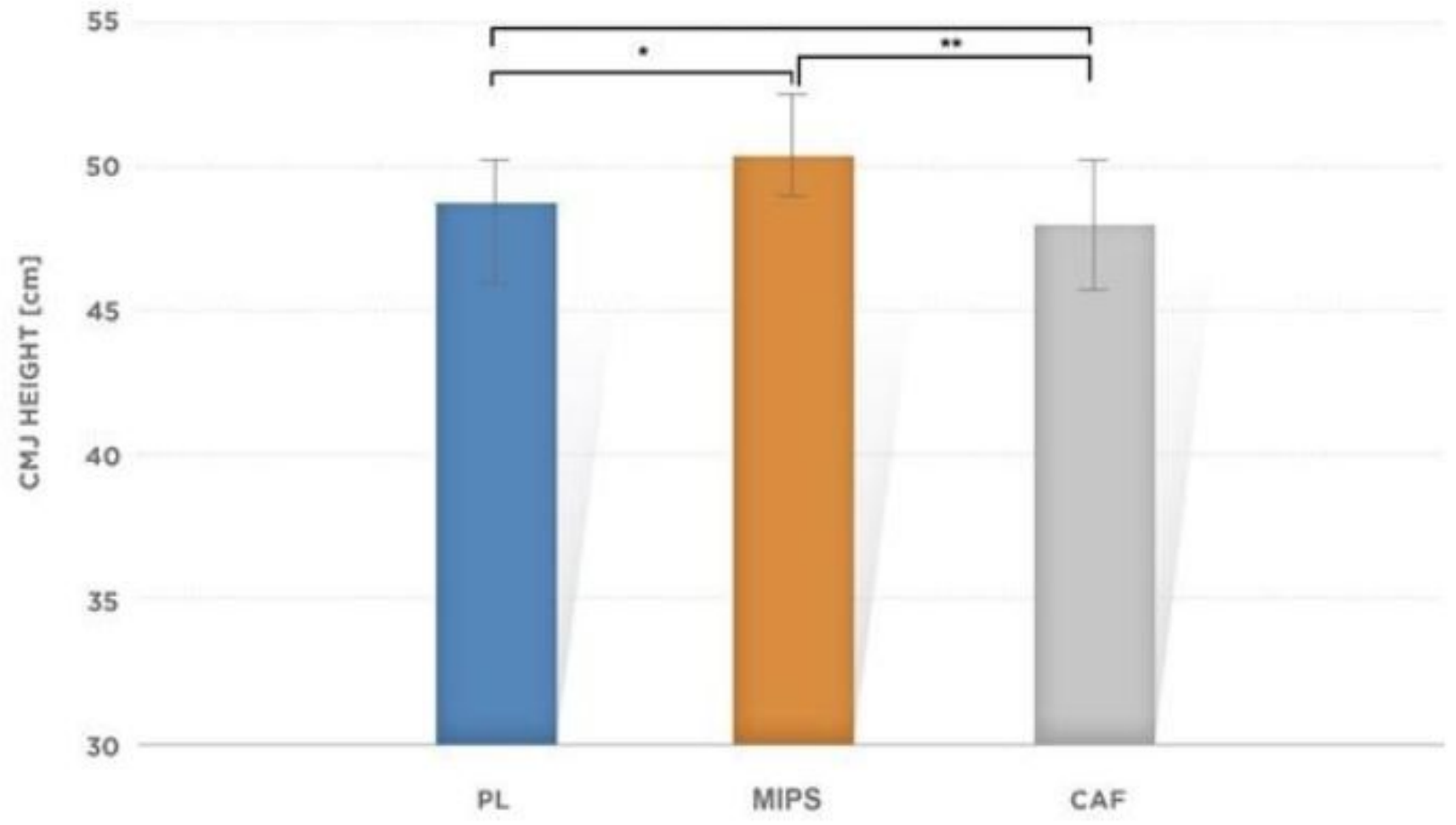

Figure 2

The mean values of countermovement jump $(\mathrm{cm})$ after PL, MIPS or CAL ingestion. Significant difference in jump height was observed between: MIPS and PL groups ( $\left.{ }^{*} \mathrm{p}=0.001\right)$ as well as between MIPS and CAF groups ( $\left.{ }^{\star *} \mathrm{p}=0.001\right)$, but not CAF and PL groups $(\mathrm{p}=0.84)$. Error bars indicate standard deviation (SD). 


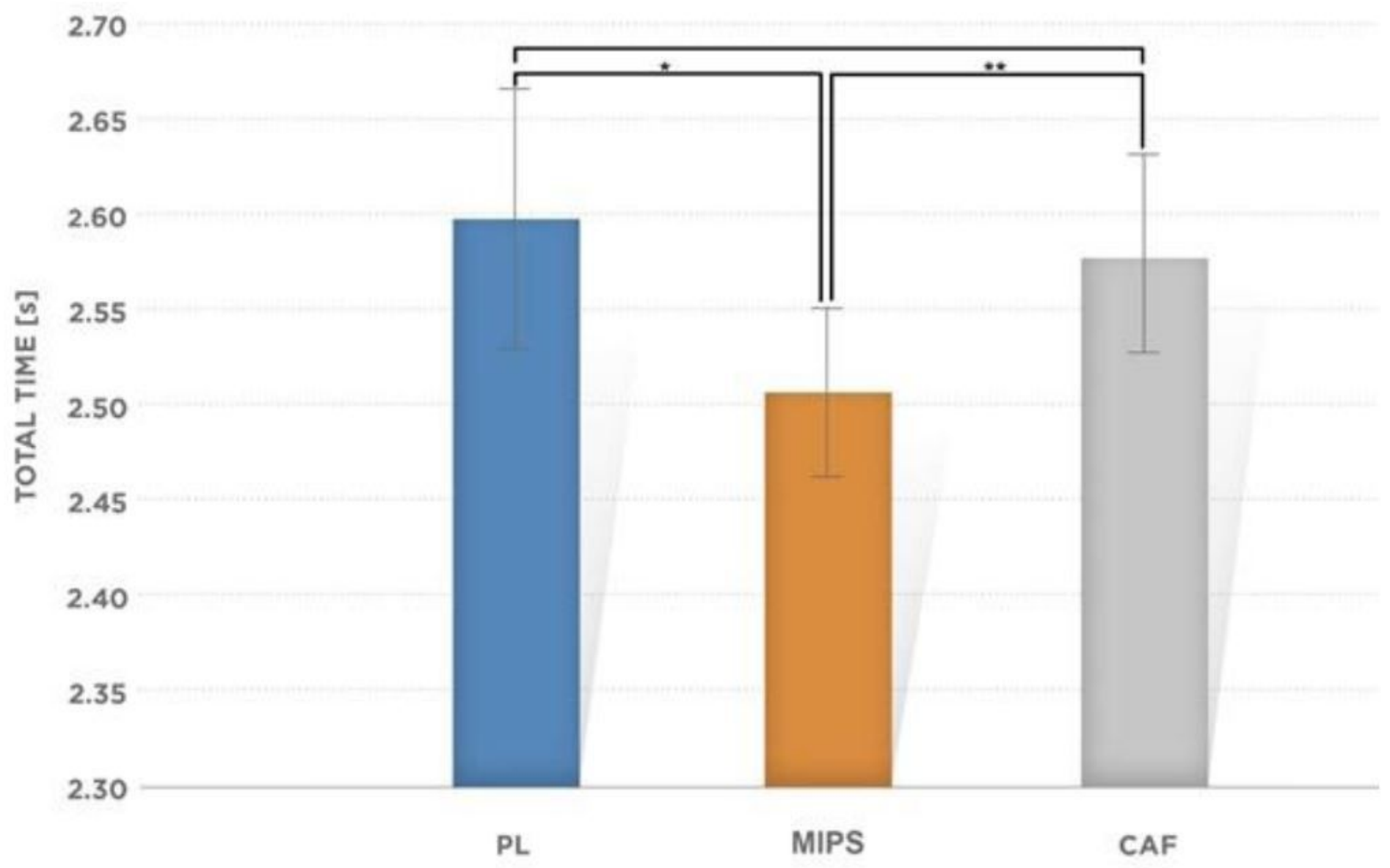

Figure 3

The mean values of total time (s) as result of 1-1-2 reactive agility test protocol after PL, MIPS or CAF ingestion. Significant difference in total time was observed between: MIPS and PL groups $\left({ }^{*} \mathrm{p}=0.02\right)$, as well as MIPS and CAF groups ( $\left.{ }^{* \star} \mathrm{p}=0.004\right)$, but not CAF and PL groups $(\mathrm{p}=0.06)$. Error bars indicate standard deviation (SD). 